\title{
Sex-Specific Associations of Body Mass Index with Mood Disturbance during Smoking Abstinence
}

\author{
Natalie A. Ceballos ${ }^{a}$ Stephanie Hooker ${ }^{b}$ Mustafa al'Absi ${ }^{\text {b-d }}$ \\ a Department of Psychology, Texas State University, San Marcos, Tex., Departments of ${ }^{b}$ Behavioral Sciences, ${ }^{c}$ Family \\ Medicine, and ${ }^{\mathrm{d}}$ Physiology and Pharmacology, University of Minnesota Medical School, Duluth, Minn., USA
}

\section{Key Words}

Body mass index $\cdot$ Nicotine $\cdot$ Smoking $\cdot$ Gender $\cdot$ Mood

\begin{abstract}
Objective: Previous reports have suggested an inverse relationship between body mass index (BMI) and negative mood in women. However, little is known about the potential association of these variables under stressful conditions, such as those experienced during smoking cessation. The current investigation examined the relationship of BMI and various indices of mood in a sample of male and female cigarette smokers undergoing cessation. Methods: Along with baseline assessments of variables such as depression and anxiety, total mood disturbance and perceived stress were assessed via self-report during both baseline (e.g. the smoking period) and the early phases of smoking cessation. Results: Among female participants, higher BMI was associated with lower levels of mood disturbance, perceived stress and depression. Similar analyses in male participants were non-significant. Conclusions: The results of the current study confirm previous reports regarding the relationship of BMI and mood in women and extend these findings to the early stages of smoking cessation.

Copyright $\odot 2009$ S. Karger AG, Basel
\end{abstract}

\section{Introduction}

For decades, scientific literature has acknowledged the 'jolly fat' hypothesis, which predicts that overweight people will have a lower risk of depression and exhibit a lower number of depressive symptoms relative to individuals of normal weight $[1,2]$. More recently, the hypothesis has resurfaced emphasizing sex differences in the relationship between obesity and mood. For instance, Oinonen and Mazmanian [3] observed an inverse relationship between body mass index (BMI) and negative mood in a sample of young female participants. The authors attributed this effect to potential increases in estrogen availability in individuals with a higher level of fatty tissues.

Although estrogen levels were not assessed in the aforementioned study, the authors' theory is worthy of further research, as estrogen is known to increase the availability of serotonin, a neurotransmitter linked with depression $[2,4]$. Thus, this mechanism may partly account for the inverse relationship noted between BMI and negative mood among females. However, it is important to acknowledge that a number of studies have reported conflicting results on this issue $[5,6]$. Further, the reason for higher estrogen levels in persons with high levels of fatty tissue would also apply to males; thus, the literature remains contentious regarding the 'jolly fat' hypothesis and a number of comorbid factors remain to be considered.

Tobacco use is one factor that has not been addressed in previous investigations of the relationship between

\section{KARGER}

(ㄷ) 2009 S. Karger AG, Basel

Fax +41613061234 E-Mail karger@karger.ch www.karger.com www.karger.com/nps
Mustafa al'Absi, $\mathrm{PhD}$

Department of Behavioral Sciences, University of Minnesota Medical School

1035 University Drive, 236 Medical School Building

Duluth, MN 55812-3031 (USA)

Tel. +1 218726 8332, Fax +1 218726 7559, E-Mail malabsi@umn.edu 
BMI and mood. Nicotine may increase energy expenditure and suppress appetite, which may account for the inverse relationship between tobacco use and body weight $[7,8]$. This finding, as well as nicotine's ability to increase the availability of serotonin in the brain [9], suggests that tobacco use may be an important consideration in studies on the connections between body mass and mood.

Smoking cessation is inherently stressful and elicits negative mood. Traditionally, smokers with a history of negative affect often have more difficulty quitting [1012]. Further, previous work has suggested that this relationship may be particularly important in women [7]. Xu et al. [13] reported that overnight nicotine abstinence produces more negative mood and craving among females than in males, contributing to the greater likelihood of females to relapse during cessation attempts. Although weight concern has been noted as a reason why women are more reluctant to quit smoking [14], no studies to date have found a relationship between BMI and length of abstinence.

The current study addresses these issues by examining several indices of mood state in both male and female participants who enrolled in a larger study of smoking cessation. Data collection was timed to examine changes in mood during the early phases of abstinence [10]. Selfreported mood state was assessed: (1) during a period when participants were smoking normally (i.e., the smoking period) and (2) in the initial phases of abstinence from nicotine (i.e., the ambulatory abstinence period).

The current study had a dual focus. First, the authors sought to replicate and extend the findings of Oinonen and Mazmanian [3] by examining the relationship between BMI and mood in a sample of male and female cigarette smokers. The authors predicted that the reported inverse relationship of BMI and mood would be apparent among female participants compared to males. These analyses focused on data from the smoking period.

A second aim of the study examined whether or not baseline BMI would predict mood alterations during the initial phase of smoking abstinence. The authors sought to determine whether or not the 'jolly fat' effect might confer some mood protection for high BMI individuals during stress (e.g. smoking cessation).

\section{Methods}

\section{Participants}

Ninety-two participants were recruited by advertisements placed in the university community as part of a larger ongoing study of stress and tobacco cessation. All participants signed con- sent forms approved by the Institutional Review Board of the University of Minnesota and received monetary incentives for their participation. Participants confirmed they had no recent history of a medical or psychiatric disorder or medication intake (except oral contraceptives), and consumed 2 or fewer alcoholic drinks per day. To meet study requirements, participants smoked a minimum of 10 cigarettes per day for at least 2 years and had a significant motivation to quit. Of those who initially qualified for the study, 20 ( 8 males) were discontinued due to failure to attend 1 or more subsequent appointments. Thus, data from 72 participants (39 males) remained. Twelve female participants from this sample reported use of oral contraceptives.

Participants began the study with the intention to schedule a specific quit date on which they would stop smoking. For female participants, the quit date was scheduled during the follicular phase (day 3-10) of the menstrual cycle, as determined by self-report. Menstrual phase at the screening session was matched to the phase at subsequent laboratory visits. In the laboratory, carbon monoxide (MicroCO; Micro Direct, Auburn, Me., USA) and salivary cotinine (EIA; DRG Diagnostics, Marburg, Germany) samples were obtained for comparison purposes to verify smoking abstinence at a later date. Prior to their quit date, participants attended a 45-min educational session focusing on the negative effects of smoking and the positive impact of cessation. No pharmacological or behavioral interventions were employed.

\section{Measures}

Background variables were assessed during the baseline laboratory visit during the smoking period. These variables included the following: age, BMI, level of nicotine dependence [15], number of cigarettes smoked per day and chronicity of tobacco use, depression, as measured via the CES-D (Center for Epidemiologic Studies Depression Scale) [16], and anxiety, as measured using the Spielberger State-Trait Anxiety Inventory [17].

The dependent variables of interest included the following assessments. The Profile of Mood States (POMS) [18] and the Perceived Stress Scale [19] were administered on 2 occasions: (1) during the smoking period, and (2) after $24 \mathrm{~h}$ of abstinence from tobacco (i.e., the ambulatory abstinence period).

The POMS consists of a set of 65 five-point adjective rating scales, with a 6-factor structure: tension-anxiety, anger-hostility, depression-dejection, vigor-activity, fatigue and confusion. A total mood disturbance score (TMD) was derived from the POMS by summing the 6 subscales with vigor weighted negatively to produce a possible range of scores from -40 to $192[18,20]$.

The Perceived Stress Scale [19] is a 14-item scale that measures the amount of stress the participants perceive in their daily lives at the time of the assessment. For the ambulatory abstinence period, the time of the last cigarette was approximately $24 \mathrm{~h}$ prior to the laboratory session. It is important to note that state measures obtained during the smoking period and ambulatory abstinence period were collected in the participants' natural environment. To screen for any anomalies in the participants' environment at the time of sample collection (e.g. traumatic incidents), information regarding the setting of data collection was documented; however, these data were not coded for analysis. Participants with significantly stressful environmental events were excluded from further study and are not included in the final sample of 72 participants included in the current analyses. 
Data Analysis

Data were analyzed using SPSS version 16. Separate t tests were used to determine potential sex differences in age, years of education, BMI, level of nicotine dependence, number of cigarettes smoked per day, chronicity of tobacco use, as well as baseline levels of anxiety and depression.

\section{Initial Correlational Analyses}

For the first focus of the study, replication/extension of the 'jolly fat' hypothesis, individual correlational analyses were conducted to determine the nature of the relationships between BMI to self-reported mood state, indexed via the POMS TMD score and perceived stress, measured using the Perceived Stress Scale. Separate analyses were conducted for males and females.

To address issues related to the interaction of sex and BMI on mood and perceived stress scores, separate median splits of BMI scores were created for males and females. BMI (high or low) and sex (male or female) were used as grouping factors in separate 2 -factorial ANOVAs with the dependent variables of TMD score and perceived stress during the smoking period.

\section{Analysis of Change Scores}

To address the secondary aim of the study, examination of the potential protective effects of BMI on mood state alterations during smoking cessation, a second set of analyses were conducted.

First, separate repeated-measures ANOVAs were conducted for males and females to examine the differences between pre(during the smoking period) and post-assessments (during the ambulatory abstinence period) for TMD and perceived stress.

Second, change scores were created by subtracting values collected at initial abstinence from values collected at the baseline assessment during the smoking period. Separate correlational analyses were used to examine the relationship between BMI and change scores for TMD and perceived stress, partialling out values collected during the smoking period. Data for males and females were analyzed separately.

Finally, separate ANCOVAs were conducted using BMI (median split: high or low) and sex (male or female) as grouping variables in the examination of change scores for TMD and perceived stress. Values of the dependent variables obtained during the smoking period were entered as covariates.

\section{Results}

\section{Background Variables}

Male and female participants were matched on age, years of education, level of nicotine dependence, number of cigarettes smoked per day, years of cigarette smoking, anxiety level, and depression level (table 1). Baseline levels of anxiety and depression were below the level of clinical significance. The BMIs of males were statistically higher overall compared to females $[\mathrm{t}(70)=2.20 ; \mathrm{p}=$ 003].

Analyses were also performed to determine relationships between BMI and factors such as anxiety and de-
Table 1. Background characteristics of male and female participants

\begin{tabular}{lcc}
\hline & $\begin{array}{l}\text { Men } \\
(\mathrm{n}=39)\end{array}$ & $\begin{array}{l}\text { Women } \\
(\mathrm{n}=33)\end{array}$ \\
\hline Age, years & $36.9 \pm 14.0$ & $34.9 \pm 14.6$ \\
Education, years & $14.3 \pm 3.0$ & $14.5 \pm 2.2$ \\
BMI* $^{*}$ & $25.9 \pm 4.0$ & $23.9 \pm 3.9$ \\
Nicotine dependence $^{1}$ & $5.6 \pm 2.1$ & $5.4 \pm 2.2$ \\
Cigarettes/day $_{\text {Smoking chronicity, years }}$ & $19.8 \pm 6.5$ & $19.1 \pm 7.7$ \\
Trait anxiety, STAI score & $14.4 \pm 2.3$ & $10.1 \pm 9.3$ \\
Depression, CES-D score & $37.2 \pm 9.0$ & $37.9 \pm 10.3$ \\
& $10.7 \pm 8.8$ & $15.1 \pm 10.2$ \\
\hline
\end{tabular}

STAI $=$ Spielberger State-Trait Anxiety Inventory. ${ }^{*} \mathrm{p}<0.05$.

${ }^{1}$ Measured by Fagerstrom Test for Nicotine Dependence.

pression. For women, BMI was negatively related to depression $[\mathrm{r}(33)=-0.46 ; \mathrm{p}=0.007]$ with a trend toward a significant correlation with anxiety $[\mathrm{r}(33)=-0.33 ; \mathrm{p}=$ $0.06]$. For men, these relationships were all highly nonsignificant [values of $\mathrm{p}>0.83$ ]. It is important to note that measures of anxiety and depression are highly related to the mood scores which were the variables of interest in the current study.

\section{Initial Correlational Analyses}

These analyses addressed the first aim of the study, that is, the replication and extension of the previous findings [3] by examining the relationship between BMI and mood in a sample of male and female cigarette smokers.

For females, BMI was significantly related to TMD score during the smoking period $[\mathrm{r}(33)=-0.42 ; \mathrm{p}=0.02]$. Women with higher BMIs reported less mood disturbance (fig. 1). Among males, no significant relationship was found between BMI and TMD $[\mathrm{r}(39)=0.09$; $\mathrm{p}=$ 0.58].

BMI was significantly related to perceived stress during the smoking period among women $[\mathrm{r}(33)=-0.35$; $\mathrm{p}=0.045]$. Women with higher BMIs reported less perceived stress (fig. 1). However, this relationship was not significant among male participants $[\mathrm{r}(39)=-0.02 ; \mathrm{p}=$ 0.89 ].

ANOVA was used as a confirmatory method for the analysis of main and interactive effects of sex and BMI group. For analyses of TMD, main effects of sex $[\mathrm{F}(1$, $68)=0.69 ; \mathrm{p}=0.41]$ and $\mathrm{BMI}$ group $[\mathrm{F}(1,68)=0.22 ; \mathrm{p}=$ $0.64]$ were not significant; however, a significant interaction of BMI group and sex was noted for TMD scores 


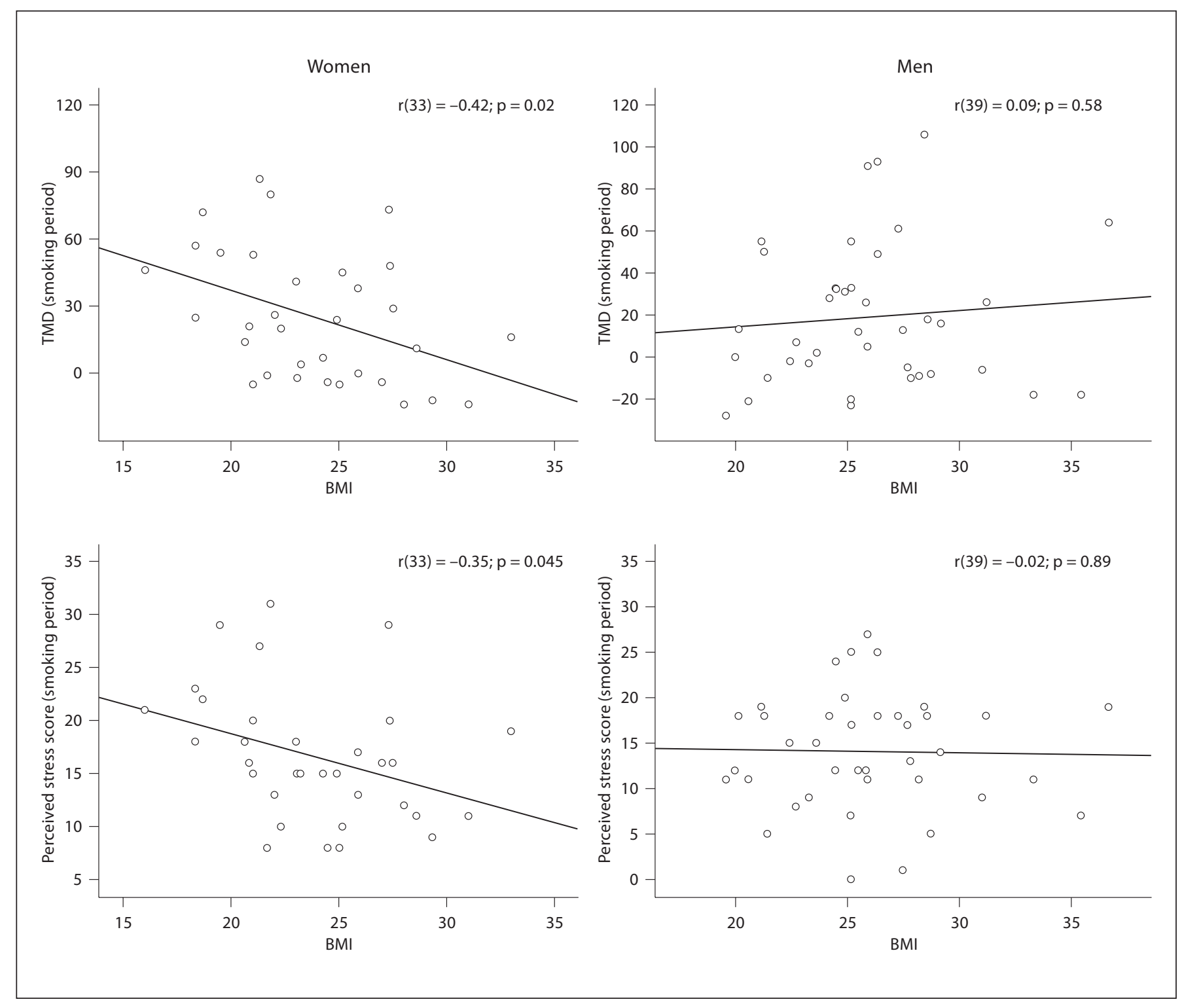

Fig. 1. TMD and perceived stress scores during the smoking period in men and women. Statistically significant relationships were noted for female, but not male, participants.

during the smoking period $[F(1,68)=5.05 ; p=0.03]$. Pairwise comparison revealed significant sex differences in TMD during the smoking period within the low BMI group $[t(34)=2.41 ; \mathrm{p}=0.02]$, in which females reported higher levels of total mood disturbance relative to males.

For analysis of perceived stress, main effects of sex $[\mathrm{F}(1,68)=2.84 ; \mathrm{p}=0.10]$, $\mathrm{BMI}$ group $(\mathrm{F}(1,68)=1.96 ; \mathrm{p}=$ $0.17)$ and their interaction $[\mathrm{F}(1,68)=2.70 ; \mathrm{p}=0.11]$ during the smoking period were non-significant.

\section{Analysis of Change Scores}

To address the second aim of the study, determining whether or not baseline BMI would predict mood alterations during the initial phase of smoking abstinence, a separate set of analyses were conducted.

First, separate repeated-measures ANOVAs were conducted for males and females to examine the differences between pre- (during the smoking period) and post-assessments (during the ambulatory abstinence period) for TMD and perceived stress. 
Women's TMD values during the smoking period (mean $=25.15, \mathrm{SD}=29.03$ ) and during the ambulatory abstinence period $($ mean $=16.09, \mathrm{SD}=36.38)$ demonstrated a trend toward a significant difference $(\mathrm{p}=0.09)$, and men's TMD values during the smoking period (mean $=19.73, \mathrm{SD}=34.47)$ and the ambulatory abstinence period $($ mean $=18.08, \mathrm{SD}=36.44)$ did not differ significantly $(\mathrm{p}=0.60)$.

The perceived stress scores of women during the smoking period (mean $=16.61, \mathrm{SD}=6.22)$ and during the ambulatory abstinence period (mean $=15.66, \mathrm{SD}=7.25)$ did not differ significantly $[\mathrm{F}(1,31)=1.18 ; \mathrm{p}=0.29]$. However, the difference between men's perceived stress values during the smoking period $($ mean $=14.08, \mathrm{SD}=6.34)$ and the ambulatory abstinence period $($ mean $=15.47, \mathrm{SD}=$ 5.93) did reach statistical significance $[\mathrm{F}(1,37)=4.19 ; \mathrm{p}=$ $0.048]$. Men perceived themselves as being significantly more stressed during the ambulatory abstinence period relative to the smoking period.

Second, separate correlational analyses partialling out values collected during the smoking period revealed a significant relationship between BMI and TMD change scores for women $[\mathrm{r}(30)=0.38 ; \mathrm{p}=0.03]$ but not for men $[r(35)=0.009 ; p=0.96]$. For measures of perceived stress change, no significant relationships were noted between perceived stress and BMI for females $[\mathrm{r}(29)=-0.15 ; \mathrm{p}=$ $0.41]$ or males $[r(35)=-0.06 ; p=0.72]$.

Finally, analysis via ANCOVA revealed nonsignificant effects of sex $[\mathrm{F}(1,66)=3.91$; $\mathrm{p}$ values $>0.13]$ on TMD change scores. However, a significant effect of BMI group was noted $[\mathrm{F}(1,66)=4.27 ; \mathrm{p}=0.04]$, in which the low $\mathrm{BMI}$ group exhibited negative change scores (i.e., stress during the smoking period was higher than stress during the ambulatory abstinence period) and the high BMI group exhibited positive change scores (i.e., stress during the smoking period was lower than stress during the ambulatory abstinence period). The interaction of BMI group and sex was also non-significant $[\mathrm{F}(1,66)=1.38 ; \mathrm{p}=$ 0.25].

Analysis of perceived stress via ANCOVA revealed non-significant effects of $\operatorname{sex}[\mathrm{F}(1,65)=2.60 ; \mathrm{p}=0.11]$, $\mathrm{BMI}$ group $[\mathrm{F}(1,65)=0.42 ; \mathrm{p}=0.52]$ and their interaction $[\mathrm{F}(1,65)=0.02 ; \mathrm{p}=0.90]$.

\section{Discussion}

The first aim of the current study was the replication and extension of the 'jolly fat' hypothesis. The current findings support this hypothesis among female partici- pants, with an inverse relationship between obesity and mood (TMD) and obesity and perceived stress during the smoking period in females, but not males (fig. 1). These relationships were investigated initially using correlational analyses, and ANOVAs were used as a confirmatory follow-up method. Given the typical increase in BMI with age, as well as the potentially increased relevance for the 'jolly fat' hypothesis in older versus younger women, additional analyses explored the relationship between BMI and mood while controlling for age. Partial correlations failed to replicate the significant relationship between BMI and mood disturbance among women during the smoking period $[\mathrm{r}(30)=0.26 ; \mathrm{p}=0.15]$. This is a particularly important point given that previous studies of the 'jolly fat' hypothesis have focused on young women [3], whereas the age range of women in the current study was quite broad (18-68 years). Thus, a number of participants in the current study may have reached menopause prior to participation in the study, an issue which could have significant implications for the hypothesized involvement of estrogen in the 'jolly fat' effect [3].

The finding of a trend toward differential sex effects in the relationship between body mass and mood disturbance is a unique addition to the literature. Further, disentangling gender differences in the 'jolly fat' phenomenon may also serve to extend and clarify previous work by Roberts et al. [21], who failed to find a significant positive association between mood and body weight in a large epidemiological study.

The mechanisms underlying this sex-specific effect are not known. However, low levels of circulating sex steroids and correspondingly low levels of the neurotransmitter serotonin have been previously associated with an increased risk of depression and/or mood disturbance [2, 7-9]. It is also important to note that serotonin is also one of several key neurotransmitter systems that play a role in nicotine withdrawal [22]. Further, although estrogen levels were not directly addressed in the current study, our data support the notion presented by Oinonen and Mazmanian [3] that increased estrogen availability in females of greater body mass may be a factor associated with decreased negative mood in female participants who have larger BMIs. Thus, one might expect to find that increased BMI and estrogen levels in women function as protective factors against the negative mood states that are often observed in the early stages of abstinence from nicotine. However, as stated previously, it is important to note that the reason for higher estrogen levels in persons with high levels of fatty tissue would also apply to males; thus, the estrogen hypothesis does not completely explain 
the presence of sex differences in the data. Additional research is needed to specifically address the potential mechanisms underlying sex differences in the relationship between BMI and mood.

Although it is clear that additional research is warranted, a recent report by Connan et al. [23] provides a relevant framework for the discussion of our findings. The authors observed increased estrogen among re-fed anorexic women and speculated that these changes were related to increased levels of leptin, a peptide hormone primarily synthesized and secreted by adipocytes [24]. These findings, although not directly related to the current study, support the notion of an interrelationship between body mass and estrogen levels among female participants.

\section{Clinical Implications}

Previous studies have shown that women often relapse to cigarette smoking after periods of abstinence for different reasons than do men $[25,26]$. One of the reasons that women frequently start smoking and find it hard to quit is because nicotine may be equated with the notion of weight loss for women; some studies have suggested that addressing issues of body image among such individuals may help to improve smoking cessation treatments [14]. Thus, the findings of the current study could conceivably be used as part of an educational program to help women deal with issues of weight and body image and the connection of these phenomena to tobacco dependence.

\section{Limitations}

Although these findings represent a significant contribution to the ongoing literature, a number of limitations remain to be addressed in future research. First, given the naturalistic design of the current study, no balanced order could be achieved between the data gathered when participants were still smoking and the data gathered after smoking cessation. As a result, the findings of the current study should be interpreted within a naturalistic framework rather than in the context of a more stringent experimental design.

Secondly, although information regarding perceived stress levels was available in the current study, specific stress levels related to smoking cessation alone were not collected. It may be argued that the difference between everyday stress before versus after smoking cessation is an indirect reflection of smoking-related stress levels, and a more sensitive measurement of smoking cessationrelated stress could be a relevant focus for future research.
The collection of additional biological samples for analysis of estrogen and leptin levels would potentially provide insight into the mechanisms underlying the 'jolly fat' effect. Via BMI, our data indirectly support the 'jolly fat' hypothesis; however, the aforementioned assays are needed to substantiate the connection between body fat, estrogen levels and mood stability among women. Further, such sensitive measures may detect subtle relationships between body fat and mood that may not reach statistical significance in analyses using height and weight. Such follow-up studies are currently underway in our laboratory.

In addition, it is well-known that participants' selfperception of body size and shape impacts mood and overall health [27], and some studies suggest that body image may play a role in suicidal ideation, particularly among adolescents [28]. Gender differences in body image are also frequently apparent $[29,30]$. The extent to which these factors may have influenced the current findings is unknown, but this remains a target of ongoing research.

Further, perceptions of body shape and size, as well as attitudes toward obesity, may be socially and culturally specific [31, 32]. For instance, being born into and/or acculturating to modern Western culture may increase the likelihood that overweight/obese individuals will internalize negative messages about their appearance, a phenomenon which may lead to depressive symptomatology [33]. As identified previously, the participant sample of the current study was predominantly born within the United States and of Caucasian origins with similar socioeconomic backgrounds. Interestingly, this participant sample composition is typical of previous studies of the 'jolly fat' hypothesis, suggesting that the factors associated with increased positive mood among women with high BMI levels is in opposition to the notion that westernized cultures internalize negative messages regarding obesity. Thus, future studies with an emphasis on the potential social and cultural influences on the interactions of body mass and mood are needed to clarify this issue, particularly among female participants.

\section{Conclusion}

Our results support and extend the 'jolly fat' hypothesis with respect to female participants undergoing the initial phases of smoking cessation. In women, but not in men, greater BMI was significantly associated with lower levels of mood disturbance. Although the mechanisms 
underlying this gender-specific effect remain to be elucidated, predictions focus on the positive relationship between BMI and subsequent levels of estrogen and serotonin. Future studies should consider more sensitive measures of body fat content and biochemical signaling as well as culturally specific differences in the perception of obesity.

\section{Acknowledgements}

The authors thank Angie Harju, Laurie Franks and Huong Timp for their assistance with data collection and management. We thank Clemens Kirschbaum of the University of Dresden, Germany, for his assistance with the project. This research was supported in part by grants from the National Cancer Institute (CA88272) and National Institute on Drug Abuse (DA013435 and DA016351).

\section{References}

1 Crisp AH, McGuiness B: Jolly fat: relationship between obesity and psychoneuroses in the general population. BMJ 1976;1:7-9.

-2 Jasienska G, Ziomkiewicz A, Gorkiewicz M, Pajak A: Body mass, depressive symptoms, and menopausal status: an examination of the 'jolly fat' hypothesis. Womens Health Issues 2005; 15:145-151.

-3 Oinonen KA, Mazmanian D: Does body fat protect against negative moods in women? Med Hypotheses 2001;57:387-388.

4 Bethea CL, Gundlah C, Streicher JM: Diverse actions of ovarian steroids in the serotonin neural system. Front Neuroendocrinol 2002;23:41-100.

5 Johnston E, Johnson S, McLeod P, Johnston $\mathrm{M}$ : The relation of body mass index to depressive symptoms. Can J Public Health 2004;95:179-183.

6 Palinkas LA, Windgard DL, Barrett-Connor E: Depressive symptoms in overweight and older adult: a test of the 'jolly fat' hypothesis. J Psychosom Res 1996;40:59-66.

7 Klein LC, Corwin EJ, Ceballos RM: Leptin, hunger, and body weight: influence of gender, tobacco smoking, and smoking abstinence. Addict Behav 2004;29:921-927.

$>8$ Klesges RC, Klesges LM, Meyers AW: Relationship of smoking status, energy balance, and body weight: analysis of the second national health and nutrition examination survey. J Consult Clin Psychol 1991;59:899905.

$\checkmark 9$ Seth P, Cheeta S, Tucci S, File SE: Nicotinicserotonergic interactions in brain and behavior. Pharmacol Biochem Behav 2002;71: 795-805.

10 al'Absi M, Hatsukami D, Davis GL, Wittmers LE: Prospective examination of effects of smoking abstinence on cortisol and withdrawal symptoms as predictors of early smoking relapse. Drug Alcohol Depend 2004;73:267-278.

-11 O’Connell KA, Martin EJ: Highly tempting situations associated with abstinence, temporary lapse, and relapse among participants in smoking cessation programs. J Consult Clin Psychol 1987;55:367-371.
12 John U, Meyer C, Rumpf HJ, Hapke U: Depressive disorders are related to nicotine dependence in the population but do not necessarily hamper smoking cessation. J Clin Psychiatry 2004;65:169-176.

13 Xu J, Azizian A, Monterosso J, Domier CP, Brody AL, London ED, Fong TW: Gender effects on mood and cigarette craving during early abstinence and resumption of smoking. Nicotine Tob Res 2008;10:1653-1661.

14 Pomerleau CS, Zucker AN, Stewart AJ: Characterizing concerns about post-cessation weight gain: results from a national survey of women smokers. Nicotine Tob Res 2001;3 51-60.

$>15$ Heatherton TF, Kozlowski LT, Freckers RC, Fagerstrom KO: The Fagerstrom Test for Nicotine Dependence: a revision of the Fagerstrom Tolerance Questionnaire. Br J Addiction 1991;86:1119-1127.

16 Radloff L: The CES-D scale: A self-report depression scale for research in the general population. Appl Psychosoc Meas 1977;1: 385-401.

17 Spielberger C, Gorsuch R, Lushene R: Statetrait Anxiety Manual. Palo Alto, Consulting Psychological Press, 1983.

18 Glover DA, Byrne J, Mills JL, Robison LL, Nicholson HS, Meadows A, Zeltzer LK: Im pact of CNS treatment on mood in adult survivors of childhood leukemia: a report from the Children's Cancer Group. J Clin Oncol 2003;21:4395-4401.

19 Cohen S, Kamarch T, Mermelstein R: A glob al measure of perceived stress. J Health Soc Behav 1983;24:385-396.

20 McNair DM, Lorr M, Droppleman LF: POMS Manual - Profile of Mood Questionnaire. San Diego, Education and Industrial Testing, 1992.

-21 Roberts R, Strawbridge WJ, Deleger S, Kaplan GA: Are the fat more jolly? Ann Behav Med 2002;24:169-180.

22 Kenny PJ, Markou A: Neurobiology of the nicotine withdrawal syndrome. Pharmacol Biochem Behav 2001;70:531-549.
23 Connan R, Lightman SL, Landau S, Wheeler M, Treasure J, Campbell IC: An investigation of hypothalamic-pituitary-adrenal axis hyperactivity in anorexia nervosa: the role of CRH and AVP. J Psychiatr Res 2006;41:131143.

24 Mukherjee R, Villarreal D, Reams GP, Freeman RH, Tchoukina I, Spear RM: Leptin as a common link to obesity and hypertension. Drugs Today 2005;41:687-695.

25 Grotvedt L, Stavem K: Association between age, gender and reasons for smoking cessation. Scand J Public Health 2005;33:72-76.

26 Westmaas JL, Langsam K: Unaided smoking cessation and predictors of failure to quit in a community sample: effects of gender. Addict Behav 2005;30:1405-1424.

27 Muennig P, Jia H, Lee R, Lubetkin E: I think therefore I am: perceived ideal weight as a determinant of health. Am J Public Health 2008;98:501-506

28 Brausch AM, Muehlenkamp JJ: Body image and suicidal ideation in adolescents. Body Image 2007;4:207-212.

$>29$ Bergeron D, Tylka TL: Support for the uniqueness of body dissatisfaction from drive for muscularity among men. Body Image 2007;4:288-295.

30 Myers TA, Crowther JH: Sociocultural pressures, thin-ideal internalization, self-objectification, and body dissatisfaction: could feminist beliefs be a moderating factor? Body Image 2007;4:296-308

31 Metcalf PA, Scragg RK, Willoughby P, Finau $S$, Tipene-Leach D: Ethnic differences in perceptions of body size in middle-aged European, Maori and Pacific people living in New Zealand. Int J Obes Relat Metab Disord 2000; 24:593-599.

$\checkmark 32$ Lynch WC, Heil DP, Wagner E, Havens MD: Ethnic differences in BMI, weight concerns, and eating behaviors: comparison of Native American, White and Hispanic adolescents. Body Image 2007;4:179-190.

-33 Schwartz MB, Brownell KD: Obesity and body image. Body Image 2004;1:43-56. 